\title{
Horizontal Well Completion with Multiple Artificial Bottom Holes Improves Production Performance in Bottom Water Reservoir
}

\author{
Haidong Wang (iD) and Yikun Liu \\ School of Petroleum and Natural Gas Engineering, Liaoning Shihua University, Fushun, China \\ Correspondence should be addressed to Haidong Wang; 565219979@qq.com
}

Received 7 August 2019; Revised 26 May 2020; Accepted 10 June 2020; Published 24 July 2020

Academic Editor: Rama S. R. Gorla

Copyright (C) 2020 Haidong Wang and Yikun Liu. This is an open access article distributed under the Creative Commons Attribution License, which permits unrestricted use, distribution, and reproduction in any medium, provided the original work is properly cited.

\begin{abstract}
The horizontal well completion with stinger is usually used to control the bottom water cone. Although the pressure profile and the inflow profile along the horizontal wellbore can be divided into two parts by the stinger, these profiles have not really flattened. In order to flatten the pressure distribution and inflow distribution further, it proposes a new technology. This new horizontal well has multiple artificial bottom holes $(\mathrm{MABH})$ along the wellbore and it has application potential. In order to verify the effectiveness of $\mathrm{MABH}$ technology, a model of horizontal well completion with $\mathrm{MABH}$ was established, and the production performance of different water cone control technologies was analyzed: conventional horizontal well, stinger completion horizontal well, and MABH completion horizontal well. The results show that the MABH technology has more advantages than the stinger technology. The uniformity of pressure distribution of the $6-\mathrm{MABH}$ horizontal well is $55 \%$ higher than that of the horizontal well with string technology, and the uniformity of inflow distribution is increased by $65.25 \%$. At the same time, although the operation of MABH technology is very simple, it should follow a rule of $\mathrm{MABH}$ installation: the position of the first MABH should be set at $242.5 \mathrm{~m}$ from the heel hole of the horizontal wellbore, and the other interval is $92.4 \mathrm{~m}$.
\end{abstract}

\section{Introduction}

The water cone is formed due to the pressure loss from the toe to the heel of the horizontal well $[1,2]$. It causes less inflow contribution from the upstream portion near the toe than from the downstream portion of the heel [3-5]. Once the bottom water breaks through into the wellbore, the water cut will rise rapidly, the production efficiency will drop significantly, and the life of the well will be greatly shortened. In order to solve this problem, some studies have been proposed as follows: the method of variable density perforation $[2,6]$ can delay the breakthrough time of water. Landman and Goldthorpe [4] established a predictive model that can calculate the inflow profile along the wellbore. In addition, the best variable density perforation technology [7] is also reported. Although this method has the effect of controlling the bottom water cone, it cannot solve the problem of pressure loss from the heel to the toe of the horizontal well.
In the early 1990s, Norwegian Hydropower engineers first proposed the concept of an inflow control device (ICD) and developed a tortuous flow control device to balance the influx of various parts along the horizontal well. Subsequently, the oil service company developed pipeline channel ICD, orifice plate ICD, automatic mixing ICD, and nozzle ICD [8-12]. Now ICD technology has been well developed. However, ICD still has some problems, such as the problem of being eroded and blocked by particles in the fluid.

On the other hand, technology of horizontal well completion with stinger was proposed [13]. It is pointed out that the stinger technology can delay the bottom water cone of long horizontal wells. The principle is to redistribute the pressure distribution of the hole by changing the actual position of the bottom hole. The optimal length of the stinger under various oil-water viscosity ratios and oil column thickness conditions were studied [5]. The stinger technology can make the wellbore pressure distribution flat, but the 
uniform effect is not ideal. Therefore, the unified problem of stinger technology should be studied in detail [14].

In this paper, a new method is proposed to further flatten the pressure profile and inflow profile along the horizontal wellbore. This method is horizontal well completion with multiple artificial bottom holes (MABH). At the same time, the pressure profile and the inflow profile along the new horizontal wellbore have been theoretically studied.

\section{Horizontal Well Completion with MABH}

In this article, we set up some independent annulus tubes through $\mathrm{MABH}$ to connect the base pipe (see Figure 1) and then use these MABH and annulus tubes to convert the unidirectional flow in the base pipe into multidirectional flow. Likewise, it is foreseeable that the pressure distribution along the base tube will exhibit periodic wavelets (red line) and that the red line will be flatter than the conventional pressure distribution (blue dotted line). Similarly, as can be seen from Figure 1, the pressure drop difference between the reservoir and the new wellbore $\left(N_{1} \approx N_{2} \approx N_{3}\right)$ is much better than the pressure drop difference between the reservoir and the conventional wellbore $\left(C_{1}<C_{2}<C_{3}\right)$. Then compare the new inflow profile $\left(N_{11} \approx N_{22} \approx N_{33}\right)$ with the traditional inflow profile $\left(C_{11}>C_{22}>C_{33}\right)$. Therefore, the heel-toe effect will be weakened.

\section{Calculation Method}

In order to explore the use of MABH technology to reduce the efficiency of the heel-toe effect, relevant theoretical calculations were carried out. The detailed calculation method is as follows.

Wellbore flow model: divide the horizontal wellbore into $N$ segments, and remove one segment for force analysis (Figure 2).

According to the law of conservation of mass, the equation can be expressed as follows:

$$
\rho_{1} A_{1} v_{1}-\rho_{2} A_{2} v_{2}+\pi D \Delta x \rho v_{r} \gamma=\frac{\partial(\rho A \Delta x)}{\partial(t)},
$$

where $\rho$ is the fluid density in the pipe, $\mathrm{kg} / \mathrm{m}^{3} ; A$ is the crosssectional area of the tube, $\mathrm{m}^{2} ; v$ is the flow velocity, $\mathrm{m} / \mathrm{s} ; \gamma$ is the opening coefficient of the base pipe; $t$ is the flow time, $s$; $\Delta x$ is the length of the infinitesimal segment, $\mathrm{m}$; and $D$ is the inner diameter of the pipe, $\mathrm{m}$.

When $\Delta x$ becomes 0 , according to the definition of derivative, equation (1) can be acquired as follows:

$$
\frac{\partial(\rho A v)}{\partial(x)}+\frac{\partial(\rho A)}{\partial(t)}=\pi D \rho v_{r} \gamma
$$

Then, according to the principle of momentum, the equation can be expressed as follows:

$$
P_{1} A_{1}-P_{2} A_{2}-\rho g A \Delta x \sin \theta-\tau_{a} \pi D \Delta x \gamma=\rho_{2} A_{2} v_{2}^{2}-\rho_{1} A_{1} v_{1}^{2}+\rho_{r} A_{r} v_{r}^{2}-\frac{\partial(v \rho A \Delta x)}{\partial(t)},
$$

where $g$ is the gravitational acceleration, $\mathrm{m} / \mathrm{s}^{2} ; \theta$ is the angle between wellbore axis and horizontal direction, degrees; and $\tau_{a}$ is the average frictional stress, $\mathrm{Pa}$.

Assuming that the flow rate is stable, and when $\Delta x$ becomes 0 , combining equations (2) and (3), the pressure drop $\Delta P_{i}$ in the base pipe can be expressed as follows:

$$
\Delta P_{i}=\rho g \sin \theta \Delta x_{i}+\zeta \frac{4 \tau_{a} \gamma}{D} \Delta x_{i}+\Omega \frac{64 \rho Q_{i} q_{r i}}{\pi^{2} D^{4} \gamma \Delta x_{i}},
$$

where $Q_{i}$ is the flux of $i$ th segment in the wellbore, $\mathrm{m}^{3} / \mathrm{s} ; q_{r i}$ is the influx rate of the $i$ th wellbore segment, $\mathrm{m}^{3} / \mathrm{s}$; $\zeta$ is the correction coefficient caused by wear and corrosion in the wellbore, dimensionless; and $\Omega$ is the correction coefficient caused by variable mass pipe flow in the wellbore, dimensionless.

Similarly, pressure drop $\Delta P_{i}$ in the annulus tube can be represented as follows:

$$
\Delta P_{i}=\rho g \sin \theta \Delta x_{i}+\zeta \frac{4 \tau_{a}}{D} \Delta x_{i} .
$$

The average frictional stress $\tau_{a}$ can be represented as follows:

$$
\tau_{a}=\frac{f \rho v^{2}}{8} .
$$

According to the research results of Ouyang [15], $f$ is shown in Table 1.
The following equation gives the friction factor:

$$
\frac{1}{\sqrt{f_{0}}}=-2 \log \left\{\frac{\varepsilon}{3.7 D}-\frac{5.02}{N_{\mathrm{Re}}} \log \left[\frac{\varepsilon}{3.7 D}-\frac{5.02}{N_{\mathrm{Re}}} \log \left(\frac{\varepsilon}{3.7 D}+\frac{13}{N_{\mathrm{Re}}}\right)\right]\right\} \text {. }
$$

Here,

$$
\begin{aligned}
N_{\mathrm{Re}, w} & =\frac{4 \rho Q_{i}}{\mu \pi D}, \\
N_{\mathrm{Re}} & =\frac{4 \rho q_{i}}{\mu \pi D},
\end{aligned}
$$

where $f_{0}$ is the correction coefficient caused by variable mass pipe flow in the wellbore, dimensionless; $N_{\mathrm{Re}}$ is the Reynolds number, dimensionless; and $\varepsilon$ is the friction factor, dimensionless.

Reservoir inflow model: firstly, we make the following assumptions: (1) The bottom water reservoir is homogeneous and infinite. (2) There is a closed boundary at the reservoir top and a constant pressure boundary at the bottom. The distance between the upper and lower boundaries is $h$, and the distance between the horizontal wellbore and the lower boundary is $z_{i}$. The reservoir temperature is constant. (3) The length of the horizontal wellbore is much larger than its diameter. (4) The model is assumed to be single-phase flow. If the pressure drops 


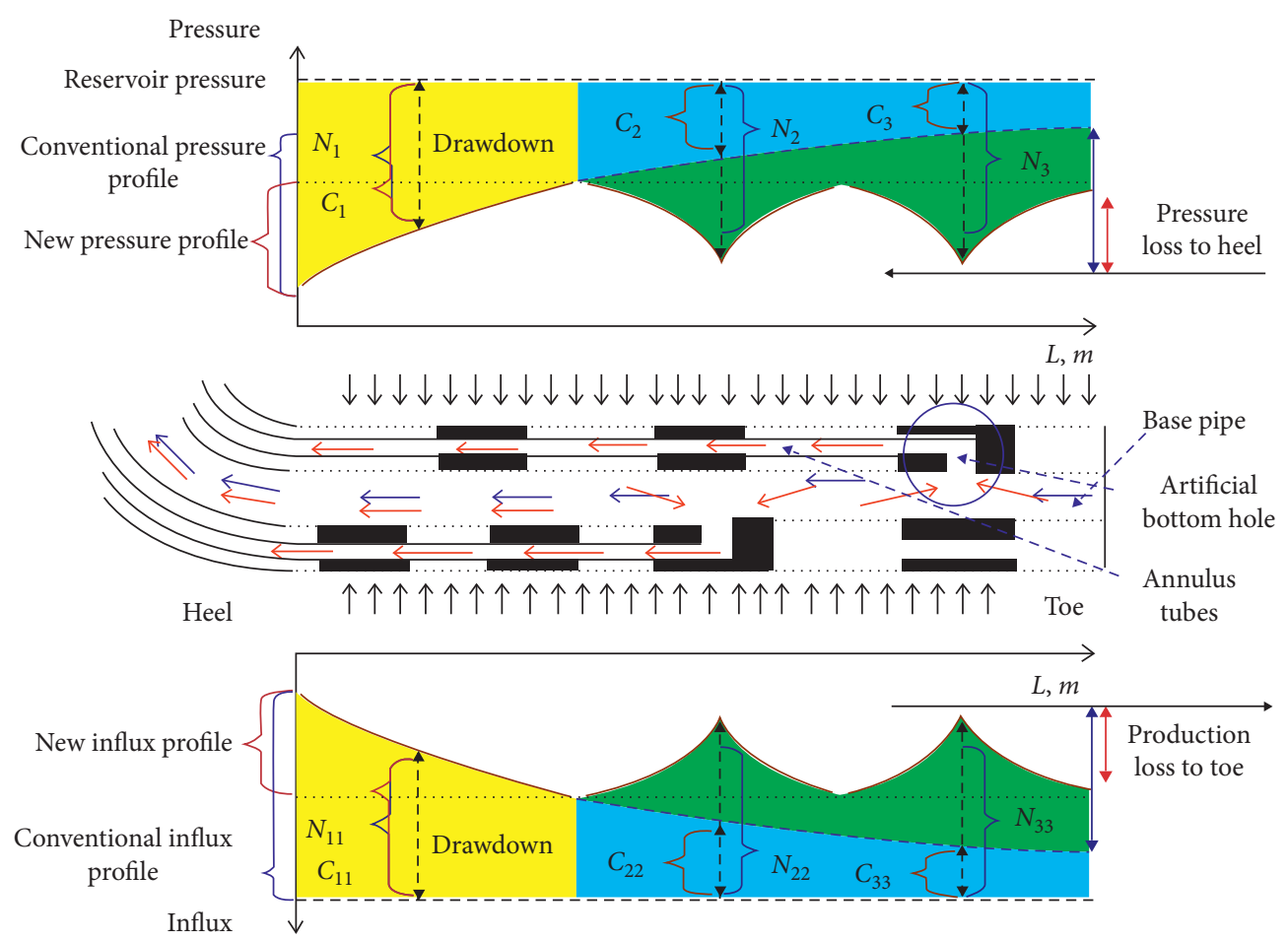

FIgURE 1: Technology of horizontal well completion with MABH.

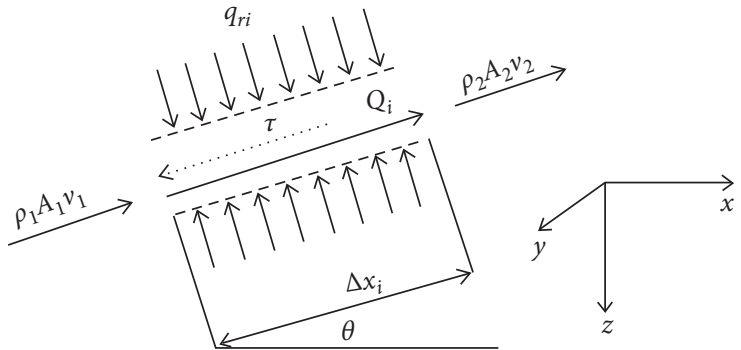

FIgURE 2: Flow analysis of the wellbore's infinitesimal section.

TABLE 1: Value of $f$.

\begin{tabular}{lcc}
\hline Flow state & Various mass flows & Constant mass flow \\
\hline Laminar & $f=f_{0}\left(1+0.043 N_{R_{e, w}}^{0.6142}\right)$ & $f=\left(16.0 / N_{R_{e}}\right)$ \\
Turbulent & $f=f_{0}\left(1+0.0153 N_{R_{e, w}^{0.3978}}\right)$ & $f^{-0.5}=\left\{-4.0 \log \left[(\varepsilon / 3.7 D)+\left(1.255 / f^{-0.5} N_{R_{e}}\right)\right]\right\}$ \\
\hline
\end{tabular}

below the bubble point, the model is not available. (5) The flow in the reservoir follows Darcy's law.

The above assumptions will affect the calculation results of the total output. However, the focus of this article is to study the performance of the inflow profile and the pressure profile of horizontal well. Therefore, these assumptions do not affect the results of this article.

First, the horizontal wellbore is divided into $N$ sections, and the penetration velocity of the $i$ th section surface can be expressed as follows:

$$
v=\frac{q_{i}}{S}=\frac{q_{i}}{2 \pi r \Delta x_{i} \gamma},
$$

where $q_{i}$ is the production rate of the $i$ th wellbore segment, $\mathrm{m}^{3} / \mathrm{s} ; S$ is the perforated opening area on the pipe, $\mathrm{m}^{2} ; v$ is the seepage velocity, $\mathrm{m} / \mathrm{s} ; S$ is the seepage area, $\mathrm{m}^{2} ; \gamma$ is the opening coefficient of the base tube; $\Delta x_{i}$ is the length of $i$ th segment, $\mathrm{m}$; and $r$ is the inner radius of the pipe, $m$.

In addition, the percolation rate can be represented as follows $[16,17]$ : 


$$
v=\left\{\begin{array}{c}
-\frac{k d_{p}}{\mu d_{r}}=\frac{d_{p}}{d_{r}}, \frac{d_{p}}{d_{r}}<\left(\frac{d_{p}}{d_{r}}\right)_{c}, \\
-k_{\text {поп }}\left(\frac{d_{p}}{d_{r}}\right)^{m}, \frac{d_{p}}{d_{r}}>\left(\frac{d_{p}}{d_{r}}\right)_{c},
\end{array}\right.
$$

where $k$ is the permeability in the reservoir, $\mathrm{m}^{2} ; \mu$ is the viscosity, $\mathrm{Pa} \cdot \mathrm{s} ; k_{\mathrm{non}}$ is nonlinear permeability coefficient near wellbore, $\mathrm{m}^{2} ;\left(d_{p} / d_{r}\right)$ is pressure gradient, $\mathrm{MPa} / \mathrm{m} ; m$ is the experimental constant, and it is between 1.2 1.5; $\left(d_{p} / d_{r}\right)_{c}$ represents the end point of linear pressure gradient, between $4 \sim 10, \mathrm{MPa} / \mathrm{m}$.
When $\left(d_{p} / d_{r}\right)<\left(d_{p} / d_{r}\right)_{c}$, the potential of the $i$ th segment in the infinite space can be obtained as follows:

$$
\varnothing=\frac{q_{i}}{2 \pi \Delta x_{i} \gamma} \ln r+C .
$$

Through the mirror image method (see Figure 3 ) and the principle of potential superposition, the potential at a random point $\beta(x, y, z)$ can be obtained as follows:

$$
\varnothing_{\beta}=\sum \varnothing_{+Q}+\sum \varnothing_{-Q} \text {. }
$$

Here,

$$
\begin{aligned}
& \sum_{n=-\infty}^{+\infty} \varnothing_{+Q}=\frac{q_{i}}{4 \pi \Delta x_{i} \gamma} \sum_{n=-\infty}^{+\infty} \ln \left[\left[\left(x-x_{i}\right)^{2}+y^{2}+\left(z-\omega_{1}\right)^{2}\right]\left[\left(x-x_{i}\right)^{2}+y^{2}+\left(z-\omega_{2}\right)^{2}\right]\right]+C \\
& \sum_{n=-\infty}^{+\infty} \varnothing_{-Q}=-\frac{q_{i}}{4 \pi \Delta x_{i} \gamma} \sum_{n=-\infty}^{+\infty} \ln \left[\left[\left(x-x_{i}\right)^{2}+y^{2}+\left(z-\delta_{1}\right)^{2}\right]\left[\left(x-x_{i}\right)^{2}+y^{2}+\left(z-\delta_{2}\right)^{2}\right]\right]+C .
\end{aligned}
$$

Furthermore, the potential of $\beta(x, y, z)$ at a random position caused by entire horizontal wellbore can be obtained as follows:

$$
\varphi_{\beta}=\sum_{n=1}^{L} \varnothing_{\beta}+C
$$

Here,

$$
\begin{aligned}
r_{i, j,+Q} & =\sqrt{\left(x-x_{i}\right)^{2}+y^{2}+\left(z-\omega_{j}\right)^{2}}, \quad j=1,2, \\
r_{i, j,-Q} & =\sqrt{\left(x-x_{i}\right)^{2}+y^{2}+\left(z-\delta_{j}\right)^{2}}, \quad j=1,2, \\
\omega_{1} & =4 n h+2 h-z_{i}, \\
\omega_{2} & =4 n h+z_{i}, \\
\delta_{1} & =4 n h+2 h+z_{i}, \\
\delta_{2} & =4 n h-z_{i}, \\
n & =0, \pm 1, \pm 2, \pm 3, \ldots, \pm \infty .
\end{aligned}
$$

Therefore, the reservoir flow in ith segment can be described as follows:

$$
P_{e}-P_{w f, i}=\sum_{n=1}^{L} \varnothing_{\beta}
$$

where $P_{e}$ is the pressure at the constant pressure boundary, $\mathrm{Pa} ; P_{w f, i}$ is the bottom hole pressure of the $i$ th segment, $\mathrm{Pa}$; $\varnothing$ is the potential, $\mathrm{Pa} ; x_{i}$ is the $x$-coordinate value of the $i$ th segment, $\mathrm{m} ; z_{i}$ is the $z$-coordinate value of the $i$ th segment, $\mathrm{m}$.

The vector representation is given by

$$
\vec{\alpha}=\vec{A} \vec{Q}
$$

Here,

$$
\begin{aligned}
\vec{\alpha} & =\left[\begin{array}{c}
P_{e}-P_{w f, 1} \\
P_{e}-P_{w f, 2} \\
\cdots \\
\cdots \\
\cdots \\
P_{e}-P_{w f, L-1} \\
P_{e}-P_{w f, L}
\end{array}\right], \\
\vec{A} & =\left[\begin{array}{c}
a_{1,1} \\
\cdots \\
\vdots \\
\ddots \\
a_{1, L} \\
\cdots \\
q_{M, i} \\
q_{1} \\
q_{2} \\
\cdots \\
\cdots \\
\cdots \\
q_{L-1} \\
q_{L}
\end{array}\right], \\
\vec{Q} & =\left[\begin{array}{c}
\varnothing_{\beta} \\
\cdots
\end{array}\right]
\end{aligned}
$$

When $\left(d_{p} / d_{r}\right)>\left(d_{p} / d_{r}\right)_{c}$, combine equations (9) and (10), flowing near the wellbore of the $i$ th segment can be described as follows:

$$
P_{e}-P_{w f, i}=\frac{m}{m-1}\left(\frac{k_{n o n} q_{i}}{2 \pi \Delta x_{i} \gamma}\right)\left[r_{e}^{(m-1 / m)}-r_{w f, i}^{(m-1 / m)}\right] .
$$

Therefore, the actual reservoir flow model is as follows: 


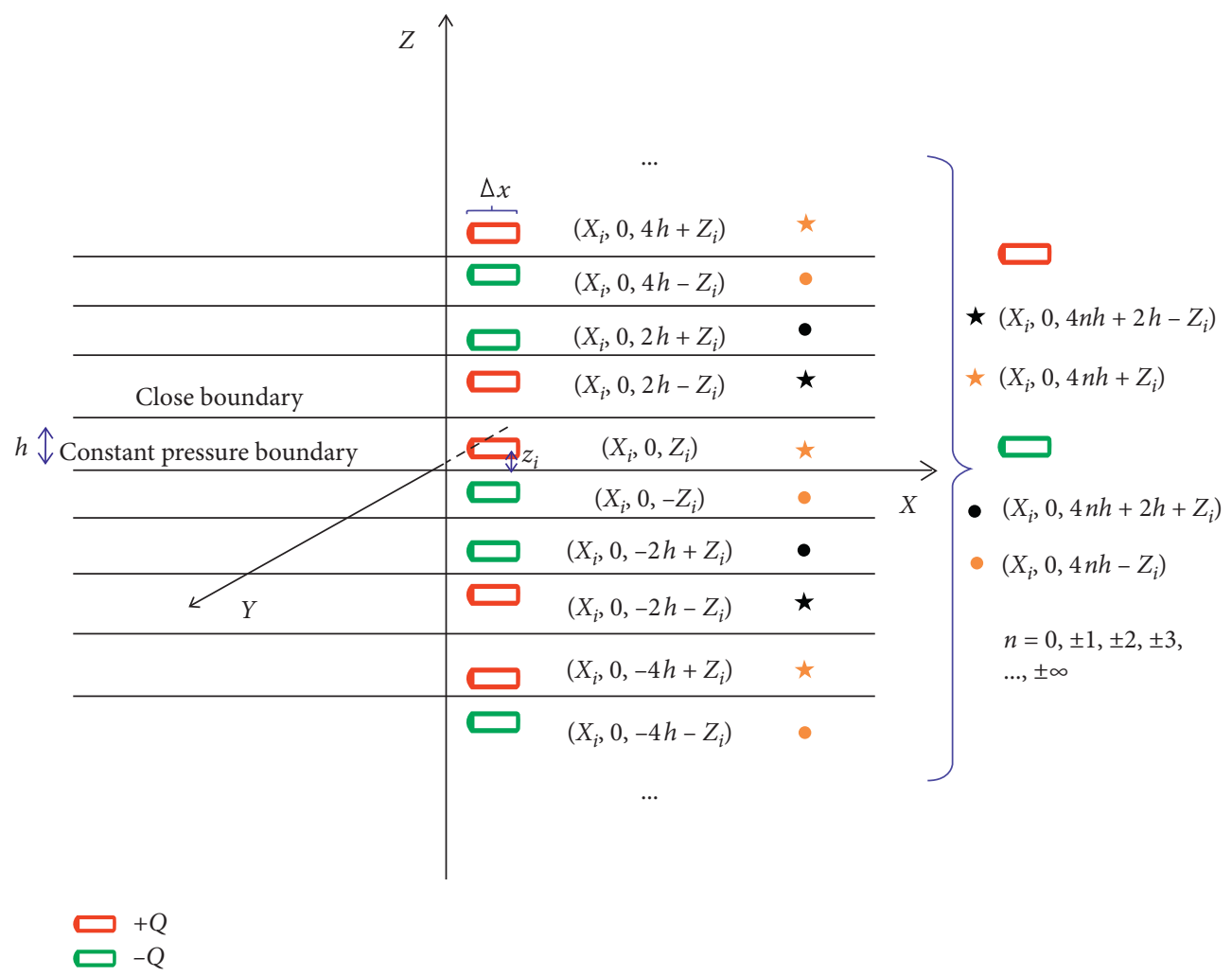

FIGURE 3: Schematic of mirror image.

$$
P_{e}-P_{w f, i}=\left\{\begin{array}{l}
\Delta \sum_{n=1}^{L} \varnothing_{\beta}, \frac{d_{p}}{d_{r}}<\left(\frac{d_{p}}{d_{r}}\right)_{c} \\
\frac{m}{m-1}\left(\frac{k_{n o n} q_{i}}{2 \pi \Delta x_{i} \gamma}\right)\left[r_{e}^{((m-1 / m))}-r_{w f, i}^{(m-1 / m)}\right] \\
\frac{d_{p}}{d_{r}}>\left(\frac{d_{p}}{d_{r}}\right)_{c}
\end{array}\right.
$$

In addition, there is oil-water flow interaction in production. Therefore, combining equations (4) and (20), the oil-water wellbore flow model and the reservoir flow model can be obtained as follows:

$$
\begin{aligned}
\Delta P_{i} & =\left(\rho_{w}+\rho_{o}\right) g \sin \theta \Delta x_{i}+\zeta \frac{4 \tau_{a} \gamma}{D} \Delta x_{i}+\Omega \frac{64 \rho\left(Q_{w, i}+Q_{o, i}\right)\left(q_{w, r i}+q_{o, r i}\right)}{\pi^{2} D^{4} \gamma \Delta x_{i}}, \\
P_{e}-P_{w f, i} & =\left\{\begin{array}{l}
\sum_{n=1}^{L} \varnothing_{\beta}, \frac{d_{p}}{d_{r}}<\left(\frac{d_{p}}{d_{r}}\right)_{c} \\
\frac{m}{m-1}\left(\frac{\left(k_{w, n o n}+k_{o, n o n}\right)\left(q_{w, i}+q_{o, i}\right)}{2 \pi \Delta x_{i} \gamma}\right)\left[r_{e}^{(m-1) / m}-r_{w f, i}^{(m-1) / m}\right], \frac{d_{p}}{d_{r}}>\left(\frac{d_{p}}{d_{r}}\right) .
\end{array}\right.
\end{aligned}
$$

where $\rho_{w}$ is the water density in the pipe, $\mathrm{kg} / \mathrm{m}^{3} ; \rho_{o}$ is the oil density in the pipe, $\mathrm{kg} / \mathrm{m}^{3} ; Q_{w, i}$ is the water flux in the $i$ th section of the wellbore, $\mathrm{m}^{3} / \mathrm{s} ; Q_{o, i}$ is the water flux in the $i$ th section of the wellbore, $\mathrm{m}^{3} / \mathrm{s} ; q_{w, r i}$ is the water inflow rate in the $i$ th wellbore segment, $\mathrm{m}^{3} / \mathrm{s} ; q_{o, r i}$ is the $i$ th water production rate of the wellbore segment, $\mathrm{m}^{3} / \mathrm{s} ; k_{w, \text { non }}$ is the nonlinear permeability coefficient of the water phase, $\mathrm{m}^{2}$; $k_{o, n o n}$ is the nonlinear permeability coefficient of the water 
phase, $\mathrm{m}^{2} ; q_{w, i}$ is the $i$ th water production rate of the wellbore segment, $\mathrm{m}^{3} / \mathrm{s} ; q_{o, i}$ is the oil production rate of the $i$ th wellbore segment, $\mathrm{m}^{3} / \mathrm{s}$.

\section{Coupling Model Solution}

The focus of this article is to study the performance of the inflow profile and pressure profile along the horizontal well. Therefore, single-phase flow or two-phase flow does not affect the results of this article and then the coupling solution takes single-phase flow as an example.

Step 1: if a set of bottom hole pressures along each $i$ th section of the horizontal wellbore are given $P_{w f, i}\left(P_{w f, 1}, P_{w f, 2}, \ldots, P_{w f, L}\right)$, the corresponding radial inflow $q_{i}(i=1,2, \ldots, L)$ can be calculated by equation (20) through the Gaussian elimination method.

Step 2: divide the horizontal wellbore into $M_{n}$ segments (Figure 4). The $M_{n}$ part of the wellbore flow will be controlled by the heel hole. Assuming that the radial inflow along the horizontal wellbore is $q_{j}\left(j=1,2, \ldots, M_{1}\right)$, the corresponding pressure difference of all pipe sections in the base pipe can be calculated by formula (4), $\Delta P_{w f, j}\left(j=1,2, \ldots, M_{1}\right)$. $Q_{M_{1}}$ is known, and the value of $Q_{M_{1}}$ can be represented as follows:

$$
Q_{M_{1}}=\sum_{j=1}^{N_{1}} q_{j}
$$

The centre pressure of the $i$ th segment of $M_{1}$ wellbore is represented as follows:

$$
P_{w f, j}^{\prime}=P_{w f,(j-0.5)}+0.5 \Delta P_{w f, j}
$$

The end pressure of the $i$ th segment of $M_{1}$ wellbore is represented as

$$
P_{w f, j+0.5}=P_{w f,(j-0.5)}+\Delta P_{w f, j},
$$

where $P_{w f, 1}^{\prime}=P_{w f \text {, heel }}$. A new group of bottom hole pressures can be obtained in section $M_{1}$, $P_{w f, j}^{\prime}\left(j=1,2, \ldots, M_{1}\right)$.

Step 3: the $M_{2}$ section is controlled by the first artificial bottom hole. In section $M_{2}$, it is divided into two parts: $M_{21}$ is the left part, and $M_{22}$ is the right part.

(1) For segment $M_{21}$, firstly assume a group of radial influx, $q_{j}\left(j=M_{1}+M_{21}-1, M_{1}+M_{21}-2, \ldots, M_{1}+\right.$ $\left.M_{21}-M_{21}\right)$. Then the corresponding differential pressure

$\Delta P_{w f, j}\left(j=M_{1}+M_{21}-1, M_{1}+M_{21}-2, \ldots, M_{1}+\right.$ $M_{21}-M_{21}$ ) of random segments in the wellbore $M_{21}$ can be calculated by equation (4).

From segment $\left(M_{1}+M_{21}\right)$ to segment $\left(M_{1}\right)$, the centre pressure of the $i$ th segment is represented as follows:

$$
P_{w f, j}^{\prime}=P_{w f,(j-0.5)}+0.5 \Delta P_{w f, j}
$$

From segment $\left(M_{1}+M_{21}\right)$ to segment $M_{1}$, the end pressure of the $i$ th segment is represented as follows:

$$
P_{w f, j+0.5}=P_{w f,(j-0.5)}+\Delta P_{w f, j}
$$

where

$$
P_{w f, M_{1}+M_{21}-1}^{\prime}=P_{\text {arwf }, M_{1}+M_{21}} .
$$

The pressure of $P_{w f, M_{1}+M_{21}}^{\prime}$ can be calculated as follows: $P_{w f, M_{1}+M_{21}}^{\prime}=P_{w f, h e e l}+\rho g \sin \theta\left(M_{1}+M_{21}\right)+\frac{4 \tau}{D}\left(M_{1}+M_{21}\right)$.

A new set of bottom hole pressures can be obtained in section $M_{21}, \quad P_{w f, j}^{\prime}\left(j=M_{1}+M_{21}-1\right.$, $\left.M_{1}+M_{21}-2, \ldots, M_{1}+M_{21}-M_{21}\right)$.

Note. If the value of $P_{w f, M 1+M 21-M 21}^{\prime}$ calculated in Step 3 is different from that of $P_{w f, M 1}^{\prime}$ calculated in Step 2, there will be two kinds of discussions:

(1) $P_{w f, M 1+M 21-M 21}^{\prime} \leq P_{w f, M 1}^{\prime}$. Part of $P_{w f, j}^{\prime}(j=$ $\left.1,2, \ldots M_{1}\right)$ in Step 2 is not accurate and it should be recalculated. Calculation should be started from segment $\left(M_{1}+M_{21}\right)$ to $\left(\left(M_{1}+M_{21}\right)-M_{21}-i\right)$ until $P_{w f,\left(M_{1}+M_{21}\right)-M_{21}-i}^{\prime}=P_{w f, N_{1}-i}^{\prime}$. Then, an accurate set of bottom hole pressures can be obtained in section $\left(M_{1}+M_{21}\right), \quad P_{w f, j}^{\prime}(j=1,2,3, \ldots$, $\left(\left(N_{1}-i\right)-1\right), M_{1}+M_{21}-M_{21}-i, M_{1}+M_{21}-$ $\left.M_{21}-i+1, \ldots, M_{1}+M_{21}\right)$.

(2) $P_{w f, M_{1}+M_{21}-M_{21}}^{\prime}>P_{w f, M 1}^{\prime}$. Part of $P_{w f, j}^{\prime}\left(j=M_{1}+\right.$ $\left.M_{21}-1, M_{1}+M_{21}-2, \ldots, M_{1}+M_{21}-M_{21}\right)$ in Step 3 is not accurate and it should be recalculated. Calculation should be started from segment $M_{1}$ to segment $\left(M_{1}+i\right)$ until $P_{w f, M_{1}+i}^{\prime}=$ $P_{w f, M_{1}+M_{21}-\left(N_{21}-i\right)}^{\prime}$. Then, an accurate set of bottom hole pressures can be obtained in section $\left(M_{1}+M_{21}\right), P_{w f, j}^{\prime}\left(j=1,2,3, \ldots, M_{1}, M_{1}+1, \ldots\right.$, $M_{1}+i,\left(M_{1}+M_{21}-\left(N_{21}-i\right)+1\right), \ldots, M_{1}+M_{21}-$ $\left.1, M_{1}+M_{21}\right)$.

(2) For segment $M_{22}$, first assume a group of radial influx, $q_{j}\left(j=M_{1}+M_{21}+1, M_{1}+M_{21}+2, \ldots, M_{1}+M_{21}+\right.$ $\left.M_{22}\right)$. The corresponding differential pressure $\Delta P_{w f, j}\left(j=M_{1}+M_{21}+1, M_{1}+M_{21}+2, \ldots, M_{1}+\right.$ $M_{21}+M_{22}$ ) of random segments in the wellbore $\mathrm{M}_{22}$ can be calculated by equation (4).

The centre pressure of the $i$ th segment of $M_{22}$ wellbore is represented as follows:

$$
P_{w f, j}^{\prime}=P_{w f,(j-0.5)}+0.5 \Delta P_{w f, j} .
$$

The end pressure of the $i$ th segment of $M_{22}$ wellbore is represented as follows:

$$
P_{w f, j+0.5}=P_{w f,(j-0.5)}+\Delta P_{w f, j},
$$




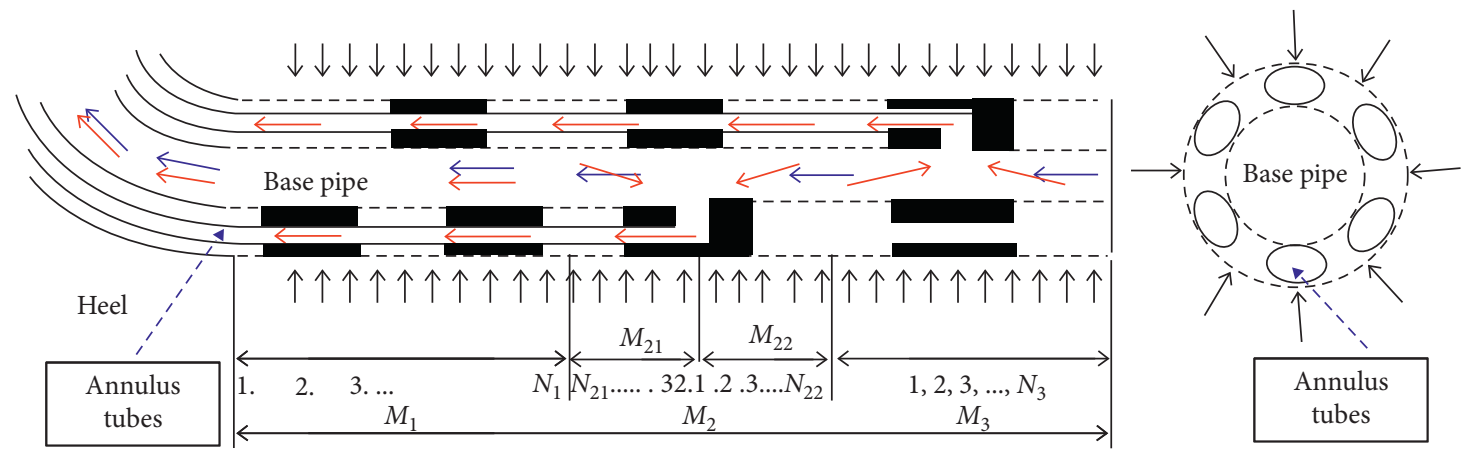

FIgURE 4: Schematic of horizontal wellbore flow with MABH.

where

$$
P_{w f, M_{1}+M_{21}}^{\prime}=P_{\text {arwf }, M_{1}+M_{21}+1}
$$

The pressure of $P_{w f, M_{1}+M_{21}}^{\prime}$ can be calculated as

$$
P_{w f, M_{1}+M_{21}}^{\prime}=P_{w f, \text { heel }}+\rho g \sin \theta\left(M_{1}+M_{21}\right)+\frac{4 \tau}{D}\left(M_{1}+M_{21}\right) \text {. }
$$

A new set of bottom hole pressures can be obtained in section $M_{22}, \quad P_{w f, j}^{\prime}\left(j=M_{1}+M_{21}+1, \quad M_{1}+M_{21}+\right.$ $\left.2, \ldots, M_{1}+M_{21}+M_{22}\right)$.

The main flow $Q_{M_{2}}$ of $M_{22}$ segment is known and can be expressed as follows:

$Q_{M_{2}}=\sum_{j=M_{1}+1}^{M_{1}+M_{21}+M_{22}} q_{j}\left(j=M_{1}+1, M_{1}+2, \ldots, M_{1}+M_{21}+M_{22}\right)$.

Step 4: repeat Step 2 and Step 3 to calculate corresponding pressure distribution controlled by the next annulus bottom hole.

Step 5: compare the pressure $P_{w f, j}$ with $P_{w f, i}$, if the inaccuracy meets the engineering inaccuracy requirement, and stop calculating or replacing Step 1 until the desired value is reached. The pressure profile and the inflow profile will be obtained.

\section{Results and Discussion}

This article focuses on a new technology to improve the impact of horizontal wellbore pressure profile and production profile, so a single-phase flow model is used. Here, we have designed three schemes (Table 2) to predict production performance. The basic data of well XU-2 is given in Table 3. The calculation method of scheme 2 is based on Permadi and Wibowo [5].

\section{Optimal Water Control Method}

Figure 5 shows three pressure distributions along the horizontal well. Here, (4), (5), and (6) represent the difference between the maximum pressure and the minimum pressure along the horizontal well (blue line). As shown in Scheme 1,
TABle 2: Schemes of different water cone controlling method.

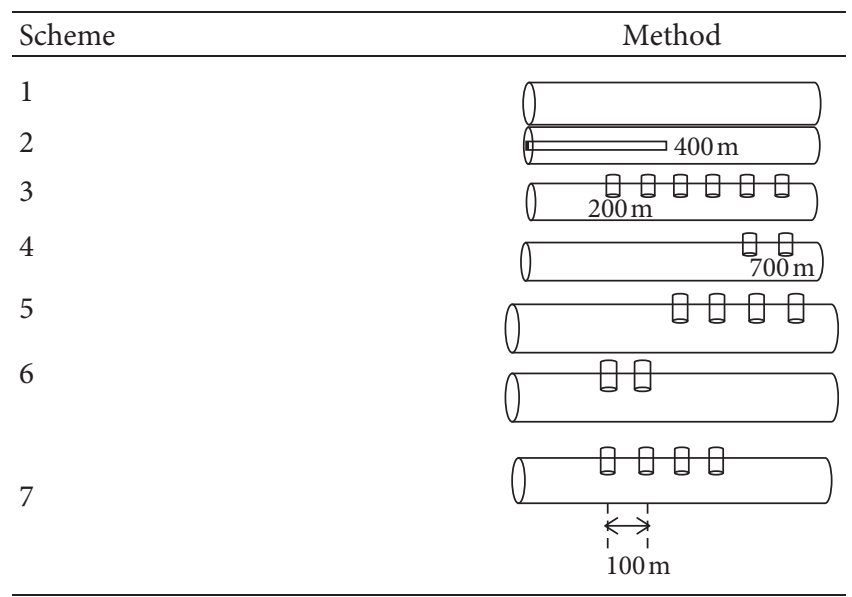

TABLE 3: Parameters related to XU-2 horizontal well.

\begin{tabular}{lc}
\hline Parameters & Value \\
\hline$P_{e}(\mathrm{MPa})$ & 12 \\
$k\left(10^{-3} \mu \mathrm{m}^{2}\right)$ & 500 \\
$L_{h}(\mathrm{~m})$ & 800 \\
$D_{\text {base }}(\mathrm{mm})$ & 100 \\
$P_{w f, \text { heel }}(\mathrm{MPa})$ & 11 \\
$\rho_{0}\left(\mathrm{~g} / \mathrm{cm}^{3}\right)$ & 0.95 \\
$\mu_{0}(\mathrm{MPa} \cdot \mathrm{s})$ & 70 \\
$h(\mathrm{~m})$ & 30 \\
$\gamma$ & 0.3 \\
$k_{r o}($ dimensionless $)$ & 0.82 \\
$D_{\text {stinger }} / D_{a}($ mm $)$ & $40 / 20$ \\
$k_{r w}($ dimensionless $)$ & 0.26 \\
\hline
\end{tabular}

the pressure distribution along the horizontal well increases monotonously, and the value of (4) is $0.0964 \mathrm{MPa}$. In Scheme 2, it can be seen that the pressure distribution along the horizontal well has been divided into two parts by the stinger and is funnel-shaped. The value of (5) is $0.0889 \mathrm{MPa}$. In Scheme 3, the pressure distribution of $\mathrm{MABH}$ horizontal wells shows wavelets, indicating that the pressure distribution of horizontal wells is divided into several wavelets using MAHB. Here, the value of (6) is only $0.04 \mathrm{MPa}$. In summary, the pressure curve of Scheme 2 is better than the 


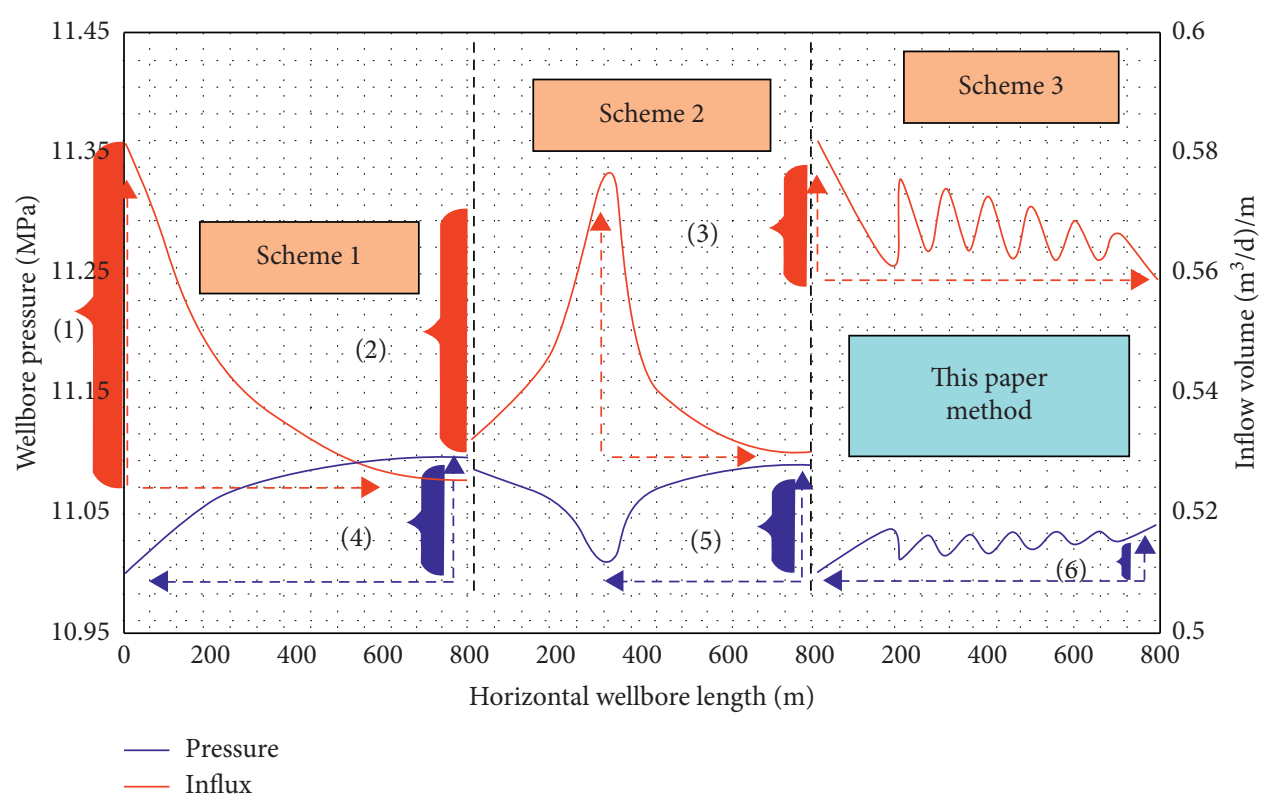

Figure 5: Pressure profile and inflow profile with different completions.

pressure curve of Scheme 1, and the uniformity of the former is $7.8 \%$ higher than that of the latter. The pressure curve in Scheme 3 is better than the pressure curve in Scheme 2, and the uniformity of the former is improved by $55 \%$ compared with the latter. Obviously, the new pressure profile of Scheme 3 is production desired.

Figure 5 also shows the distribution of inflow profiles along horizontal wells. Here, (1), (2), and (3) represent the difference between the maximum inflow and the minimum inflow along the horizontal wellbore, respectively. As can be seen from Scheme 1, the value of $(1)$ is $0.0561\left(\mathrm{~m}^{3} / \mathrm{d}\right) / \mathrm{m}$. It can be seen from Scheme 2 that the difference can be reduced by using a stinger, but the inflow fluctuation range between the funnel point and the end of the wellbore is still very large, and the value of (2) is $0.0468\left(\mathrm{~m}^{3} / \mathrm{d}\right) /$ meter. It is worth noting that in Scheme 3, by using $\mathrm{MABH}$, the value of (3) is only $0.01626\left(\mathrm{~m}^{3} / \mathrm{d}\right) / \mathrm{m}$. Compared with (3) and (2), the uniformity of the former is improved by $65.25 \%$ than the latter. Therefore, it can be concluded that MABH can further flatten the inflow profile.

\section{Optimal Installation Interval of MABH}

Figure 6 shows the pressure profile and inflow profile. The inflow profile gradually flattens and periodically flattens as the number of $\mathrm{MABH}$ increases. In addition, when the same amount of $\mathrm{MABH}$ is installed at the toes, the uniformity of the pressure profile and the inflow profile will be more uniform than the installation at the heel. When the length of the base pipe controlled by the wellbore hole is less than $242.5 \mathrm{~m}$ and the length of the MABH controlled base pipe is less than $92.4 \mathrm{~m}$, the difference between the maximum inflow and the minimum inflow along the horizontal wellbore does not exceed 3\% of the average value. Therefore, we set the first $\mathrm{MABH}$ to $242.5 \mathrm{~m}$ along the horizontal well, and other installation intervals to $92.4 \mathrm{~m}$. But this rule only applies to wellbore with the above parameters. For other wellbores, we can recalculate the optimal length of the base pipe controlled by $\mathrm{MABH}$ according to the above method.

\section{Operation and Challenges}

The annulus tube can be oval and can be extruded through a seamless steel tube. It is locked between the base pipe and the screen pipe by the support ring and crossover. Here, you can set a $\mathrm{MABH}$ on the cross ring and connect the $\mathrm{MABH}$ to the annulus and the base pipe (see Figures 1 and $7(\mathrm{e})$ ), respectively. Then, Figures $7(\mathrm{a})-7(\mathrm{e})$ show the ground installation process of the production unit with $\mathrm{MABH}$. Install the screen spacers, wire wrap screen, crossover with $\mathrm{MABH}$ and support rings, annulus tubes, and screen shroud at the corresponding positions of the base pipe until the production unit shown in Step 6 is formed. Then, it is possible to form horizontal wells with $\mathrm{MABH}$ by connecting each production unit in accordance with the steps given in Figures $7(\mathrm{~g}), 7(\mathrm{~h})$, and $7(\mathrm{i})$. In particular, after tightening the connection of the base pipe, annulus tubes are automatically further inserted upstream of annulus tubes on both sides, which has a good sealing and self-locking function. The completion structure of $\mathrm{MABH}$ horizontal well is complicated, but the operation is simple and convenient. Similarly, when the length of the annulus tube is designed to be $10 \mathrm{~m}$ and the cross section is designed to be $314 \mathrm{~mm}^{2}$, the cost of an annulus tube is about \$ 610. This is the challenge of developing low-yield fields. But it is suitable for high-yield bottom water oil fields or gas fields.

\section{Further Discussion}

The reason for the multidirectional flow in the base pipe can be obtained from the following equation: 


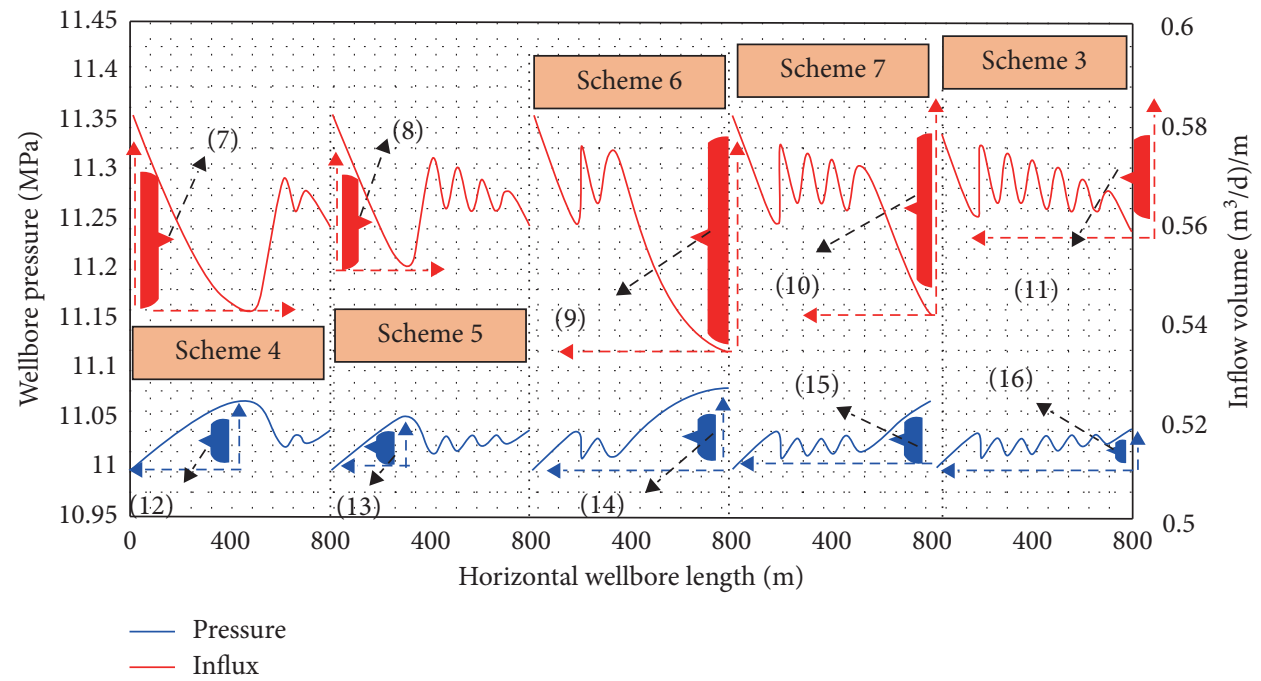

FIgUre 6: Pressure profile and inflow profile with different installation conditions.

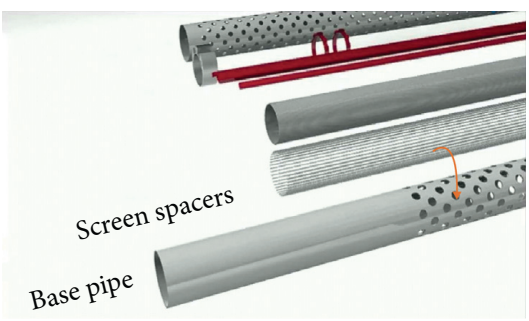

(a)

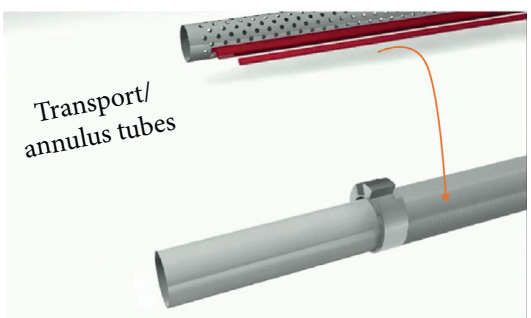

(d)

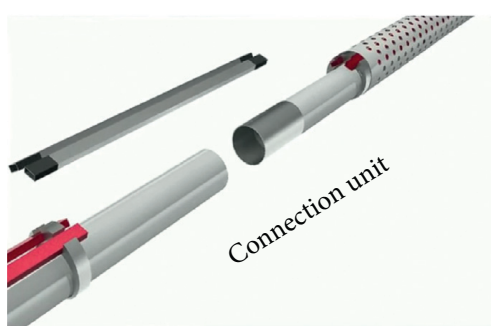

(g)

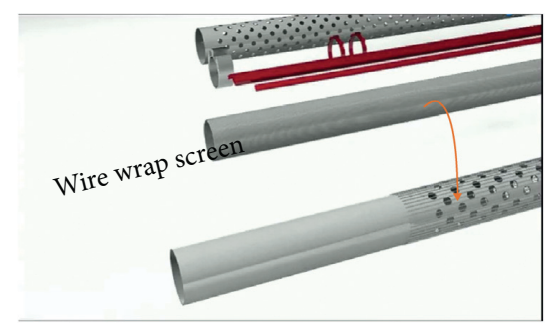

(b)

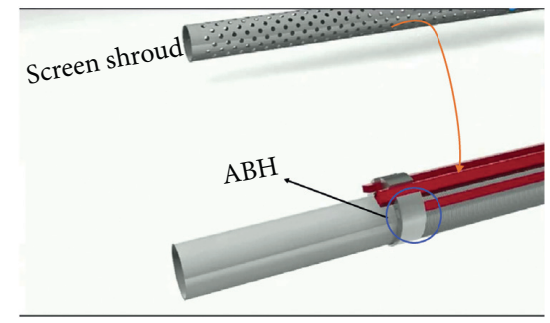

(e)

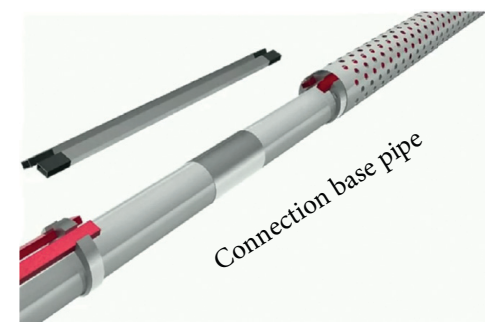

(h)

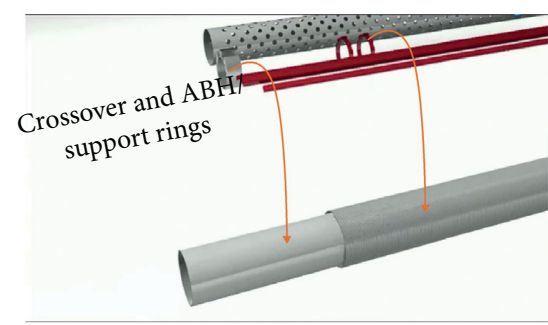

(c)

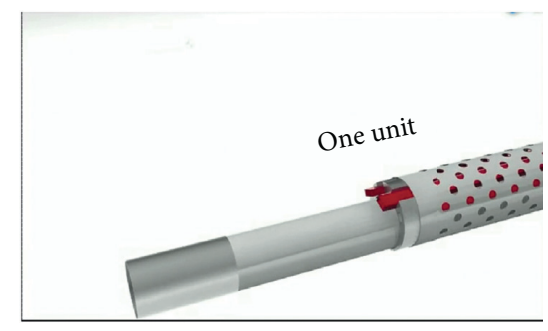

(f)

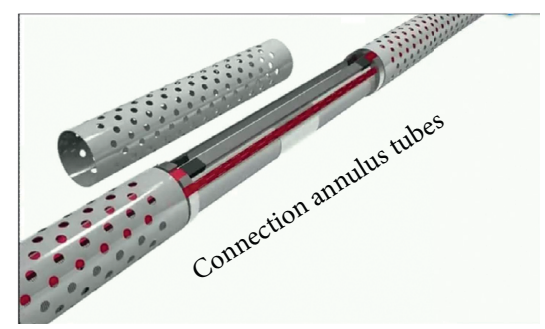

(i)

FIgURE 7: Operation process of horizontal well completion with MABH.

$\Delta P=\left\{\begin{array}{l}\Delta P_{g}+\Delta P_{s}+\Delta P_{a c c}, \text { various mass flows (base pipe flow) }, \\ \Delta P_{g}+\Delta P_{s}, \text { constant mass flow (annulus tube flow), }\end{array}\right.$

where $\Delta P$ is the pressure drop along the wellbore, $\mathrm{MPa} ; \Delta P_{g}$ is the gravity pressure drop, $\mathrm{MPa} ; \Delta P_{s}$ is the friction pressure drop, $\mathrm{MPa}$; and $\Delta P_{\text {acc }}$ is the acceleration pressure drop, $\mathrm{MPa}$.
The results show that, compared with the constant mass flow in the annulus tube, the various mass flows in the base pipe overcome the greater flow resistance. The reason is because there are perforated openings in the base pipe, but not in the annulus tube, so the additional $\Delta P_{a c c}$ in the base pipe will be formed by radial inflow acceleration. Therefore, the lower pressure in the base pipe at the toe can be controlled by using $\mathrm{MABH}$, and then the fluid at the toe can 


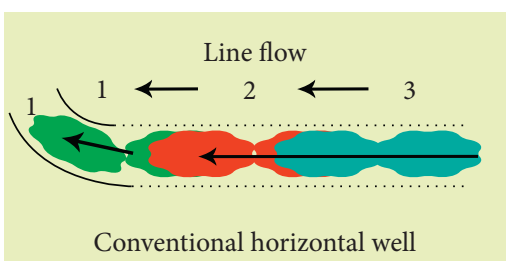

(a)

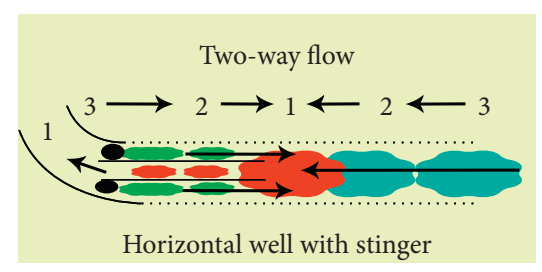

(b)

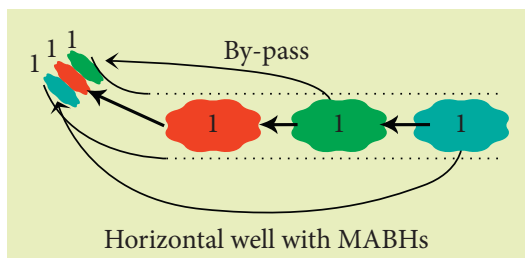

(c)

Figure 8: MABH technical mechanism.

easily bypass the heel (as shown in Figures 1 and 8). The influx of toes in the wellbore has increased, which is beneficial for reducing the heel-toe effect of horizontal wells. In short, MABH technology should be considered when controlling the water cone problem of horizontal wells for better reservoir management.

\section{Conclusions}

Here are some suggestions and conclusions:

(1) A new MABH completion method is established to solve the water coning problem. The main mechanism of MABH technology is to reduce the original pressure in the conventional horizontal wellbore by using $\mathrm{MABH}$. The original streamline from the toe to the heel in a conventional horizontal wellbore is changed by multidirectional flow. Then, the pressure distribution along the base pipe can be flattened, and the inflow profile along the horizontal wellbore can be made uniform.

(2) A coupling model of horizontal well with MABH and reservoir is proposed, which can be used to calculate the pressure loss and inflow difference along the horizontal well. Then, the dynamics of water control technologies are compared based on the output. The influence of the type of wellbore on the development effect is also discussed. Both methods can effectively control the pressure profile and the inflow profile, but the system with 6MABH has significantly improved the pressure profile and the inflow profile than the system with stinger. The uniformity of the pressure distribution of the horizontal well using $6-\mathrm{MABH}$ is $55 \%$ higher than that of the horizontal well using the stinger, and the uniformity of the inflow profile is increased by $65.25 \%$.

(3) Although the MABH structure is complex, the operation is simple and convenient. The cost of a $\mathrm{MABH}$ and an annulus tube with a length of $10 \mathrm{~m}$ and a cross section of $314 \mathrm{~mm}^{2}$ is only $\$ 610$, which is a challenge for the development of low-yield oil fields. At the same time, the first MABH should be set at $242.5 \mathrm{~m}$ away from the heel hole of the horizontal wellbore, and the other interval is $92.4 \mathrm{~m}$.

\section{Data Availability}

The .xlsx data used to support the findings of this study are included within the article and can be downloaded from
https://pan.baidu.com/s/1Bj6Ld1EHi4eH62N4200Pcg, and the supporting data extraction code is p7s1.

\section{Conflicts of Interest}

The authors declare no conflicts of interest.

\section{Acknowledgments}

This study was supported by Major Project of China National Offshore Oil Corporation 13th five-year plan (Grant no. CCL2018ZJFN0462).

\section{References}

[1] B. P. Marett and M. J. Landman, "Optimal perforation design for horizontal wells in reservoirs with boundaries," in Proceedings of the SPE Asia Pacific Oil and Gas Conference, Singapore, February 1993.

[2] H. J. Yuan, CemSarica, and J. P. Brill, "Effect of perforation density on single-phase liquid flow behavior in horizontal well," SPE Production \& Facilities, vol. 14, 1999.

[3] I. Stanley Okafor, C. Ebenezer Ubani, and N. Anyanwu, "Development strategies for oil reservoirs with coning problems: a simulation case study," in Proceedings of the SPE Nigeria Annual International Conference and Exhibition, Lagos, Nigeria, August 2018.

[4] M. J. Landman and W. H. Goldthorpe, "Optimization of perforation distribution for horizontal wells," in Proceedings of the SPE Asia-Pacific Conference, Perth, Australia, November 1991.

[5] P. Permadi and W. Wibowo, "Horizontal well completion with stinger for reducing water coning problems," in Proceedings of the SPE Production Operations Symposium, Oklahoma City, OK, USA, March 1997.

[6] T. Yildiz, "Inflow performance relationship for perforated horizontal wells," in Proceedings of the SPE Production and Operations Symposium, Oklahoma City, OK, USA, March 2001.

[7] T. Yildiz, "Productivity of selectively perforated horizontal wells," in Proceedings of the SPE Production \& Operations, Oklahoma City, OK, USA, February 2006.

[8] A. B. Elverh $\phi y$ and H. Aakre, "Autonomous inflow control for reduced water cut and/or gas oil ratio," in Proceedings of the Offshore Technology Conference, Houston, TX, USA, May 2018.

[9] D. Zubarev, E. Nnebocha, and A. Vasper, "Feasibility study and selection process for icd applications in an offshore niger delta field," in Proceedings of the SPE Nigeria Annual International Conference and Exhibition, Lagos, Nigeria, August 2018. 
[10] D. C. Haeberle, A. Nwankwo, J. R. Mcdermott et al., "Application of flow-control devices for water injection in the erha field," in Proceedings of the IADC/SPE Drilling Conference, Orlando, Florida, USA, March 2008.

[11] C. Jones, Q. Morgan, S. Beare et al., "Design, testing, qualification and application of orifice type inflow control devices," in Proceedings of the International Petroleum Technology Conference, Doha, Qatar, December 2009.

[12] B. Least, S. Greci, and R. Huffer, "Steam flow tests for comparing performance of nozzle, tube, and fluidic diode autonomous ICDs in SAGD wells," in Proceedings of the SPE Heavy Oil Conference-Canada, Calgary, Canada, June 2014.

[13] K. Brekke and S. C. Lien, "New and simple completion methods for horizontal wells improve production performance in high-permeability, thin oil zones," SPE Drilling \& Completion, vol. 9, 1994.

[14] H. Li, B. Jiang, and Y. Wang, "A semianalytical model for horizontal wells with improved stinger completion in heterogeneous bottomwater reservoirs," Journal of Canadian Petroleum Technology, vol. 53, 2014.

[15] L.-B. Ouyang, Single phase and multiphase fluid flow in horizontal wells, Ph.D. thesis, Stanford University, Stanford, CA, USA, 1998.

[16] S. Hansbo, "Consolidation equation valid for both Darcian and non-Darcian flow," Géotechnique, vol. 51, no. 1, pp. 51-54, 2001.

[17] S. Hansbo, "Deviation from Darcy's law observed in onedimensional consolidation," Géotechnique, vol. 53, no. 6, pp. 601-605, 2003. 